\title{
Rapid magnitude determination from peak amplitudes at local stations
}

\author{
Akio Katsumata ${ }^{1}$, Hiroshi Ueno ${ }^{1}$, Shigeki Aoki ${ }^{1}$, Yasuhiro Yoshida ${ }^{2}$, and Sergio Barrientos ${ }^{3}$ \\ ${ }^{1}$ Meteorological Research Institute, JMA, 1-1 Nagamine, Tsukuba, Ibaraki 305-0052, Japan \\ ${ }^{2}$ Ministry of Education, Culture, Sports, Science and Technology in Japan, 3-2-2 Kasumigaseki, Chiyoda-ku, Tokyo 100-8959, Japan \\ ${ }^{3}$ Departamento de Geofisica, Universidad de Chile, Blanco Encalada 2002, Santiago, Chile
}

(Received August 9, 2012; Revised March 12, 2013; Accepted March 12, 2013; Online published September 17, 2013)

\begin{abstract}
The rapid determination of its magnitude soon after a great earthquake is necessary for the issuing of effective tsunami warnings, as demonstrated in the great earthquake off Tohoku district in Japan on March 11, 2011. The earthquake magnitude for the first tsunami warning was underestimated due to magnitude saturation. This paper proposes a method to determine magnitude rapidly from peak velocity and displacement of long-period seismic waves up to 100 seconds at local stations. When waveform data at local stations are available, the magnitude from $S$-wave peaks is expected to be determined faster than that from only $P$-wave peaks. It takes about 140 seconds to estimate a magnitude of about 9 for the March 11, 2011, earthquake, which would enable us to issue the first tsunami warning within three minutes after the same type of earthquake.
\end{abstract}

Key words: Magnitude determination, tsunami warning, long-period seismic wave, great earthquakes.

\section{Introduction}

The displacement magnitude determined by the Japan Meteorological Agency (JMA) (Katsumata, 2004) indicated saturation during the 2011 off the Pacific coast of Tohoku Earthquake on March 11 of $M_{\mathrm{w}} 9.0$ (Hirose et al., 2011). Magnitude determination is a key to issuing an effective tsunami warning. The JMA displacement magnitude is determined from the logarithm of the maximum displacement amplitude recorded with seismographs of natural period $6 \mathrm{~s}$ and damping coefficient of 0.55. Displacement records are currently obtained from acceleration records by numerical integration and digital filtering. Using longerperiod seismic waves for magnitude determination should overcome the problem of magnitude saturation (Aki, 1967). Here, we use the peak velocity and displacement of a longer period than that used for the JMA magnitude to rapidly determine the magnitude.

Several magnitude determination methods have been proposed for a tsunami warning based on $P$-wave before the $S$-wave arrival (Tsuboi et al., 1995; Yoshida, 1995; Hara, 2007; Kanamori and Rivera, 2008; Lomax and Michelini, 2009). When the fault rupture lasts a long time, the epicentral distance of data should be great enough to get a $T_{S}-T_{P}$ exceeding the rupture duration, where $T_{P}$ and $T_{S}$ are the travel times of $P$ and $S$ waves.

The broken curve in Fig. 1 indicates the time when the $P$-wave magnitude can be determined. The time is considered to be the sum of the $P$-wave travel time and the source duration of the earthquake. The source duration of an earthquake is assumed to be sixty seconds in the figure. Since

Copyright (c) The Society of Geomagnetism and Earth, Planetary and Space Sciences (SGEPSS); The Seismological Society of Japan; The Volcanological Society of Japan; The Geodetic Society of Japan; The Japanese Society for Planetary Sciences; TERRAPUB.

doi:10.5047/eps.2013.03.006 the $S$-wave amplitude often exceeds the $P$-wave amplitude, the $P$-wave magnitude should be determined from the data before the $S$-arrival. The $T_{S}-T_{P}$ time should exceed the source duration $(D)$ for the $P$-wave magnitude. The range of $T_{S}-T_{P}<D$ is not shown in the figure. The solid curve in the figure denotes $T_{S}+D$, which is the time when the $S$-wave magnitude can be determined. When data at local stations are available, the magnitude can be determined more quickly from the amplitude of $S$-waves than from that of only $P$-waves.

Quick magnitude determination methods have been proposed also for early earthquake warning (Wu et al., 1998; Kamigaichi, 2004; Wu and Zhao, 2006; Zollo et al., 2006). However, those methods are based on the amplitude measured on waveforms of intermediate period, or the amplitude of the initial parts after the onsets, and do not fit magnitude determination for great and long source duration earthquakes.

For the $P$-wave magnitude determination, it is necessary to restrict the amplitude search range within $P$ - and $S$-arrivals to avoid $S$-wave contamination. When any phase type indicating the peak amplitude can be used, time windows for the amplitude search are not needed. This makes the process flow simple and robust. Here, we examine the magnitude determined from the peak amplitude of any phases including long-period $S$-waves and surface waves.

\section{Method and Data}

The peak velocity (m/s) or displacement (m) $A$ and magnitude $M$ are assumed to be expressed as follows:

$$
M=a \log _{10} A+b \log _{10} R+c .
$$

Here, $a, b$, and $c$ are constants, and $R(\mathrm{~km})$ is the hypocentral distance. $A$ is measured over a seismic record on a vertical component obtained at a local station. A dip slip along 


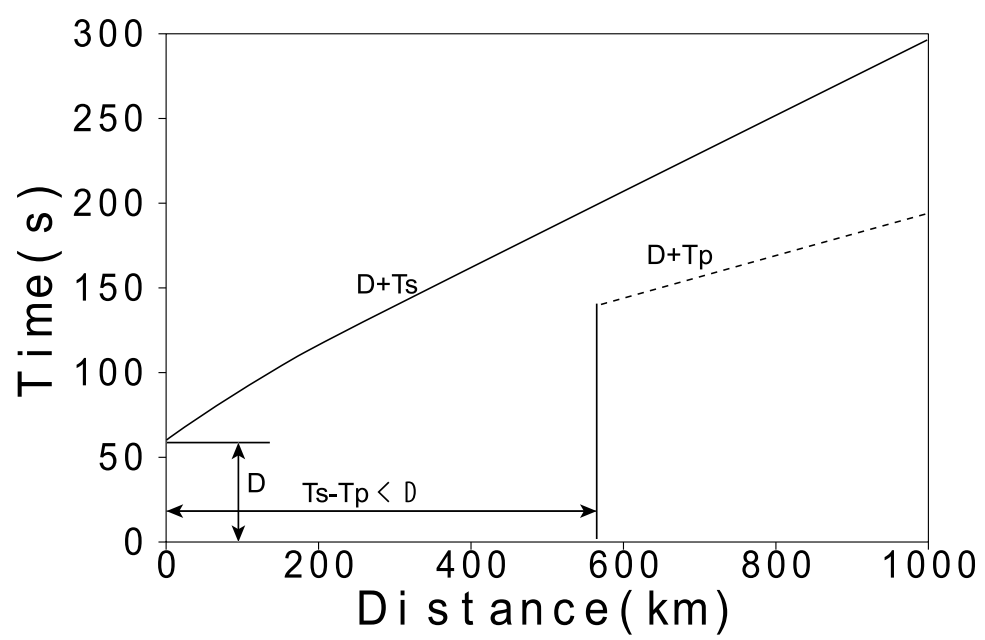

Fig. 1. Assumed time to determine earthquake magnitude from $P$-wave (the broken curve) and from $S$-wave (the solid curve). The curves indicate relationships between the epicentral distance and the sums of travel times $\left(T_{P}, T_{S}\right)$ and the assumed rupture duration $(D)$. The rupture duration is assumed to be sixty seconds.

$\mathrm{T} \mathbf{c}=1(\mathrm{~s})$

$\mathbf{T} \mathbf{c}=2(\mathrm{~s})$

$\mathrm{T} \mathbf{c}=5(\mathrm{~s})$

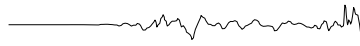

$\mathrm{Tc}=\mathbf{1 0}(\mathrm{s})$

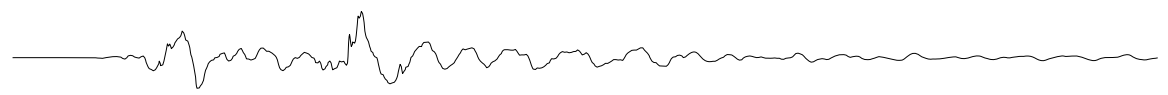

$\mathrm{Tc}=\mathbf{2} \mathbf{0}(\mathrm{s})$

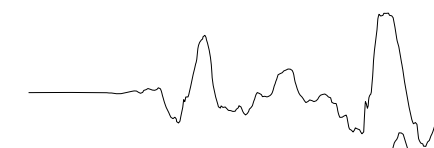

$\mathrm{Tc}=\mathbf{5 0}(\mathrm{s})$
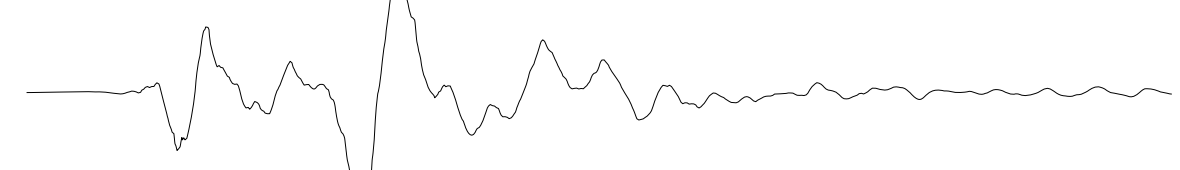

$\mathrm{Tc}=100(\mathrm{~s})$
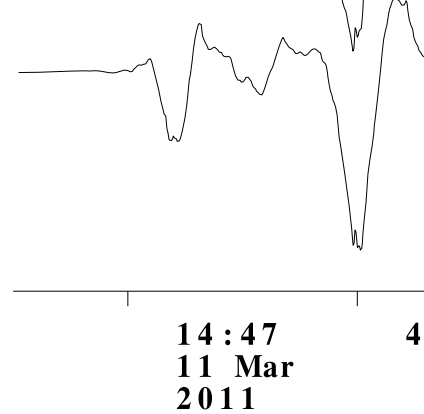

48

49

50

51

Fig. 2. Examples of waveform data used in this study. Filters of various cutoff periods $\left(T_{c}\right)$ are used to obtain the displacement records. The waveform was obtained for the 2011 off the Pacific coast of Tohoku Earthquake on March 11 at an epicentral distance of $319 \mathrm{~km}$.

a plate boundary may cause a large tsunami, and is expected to generate large $P-S V$ mode seismic waves, which appear on the vertical component.

Velocity and displacement records are obtained from strong-motion acceleration records with numerical integra- tion and low-cut filters. Second- (for velocity) and Third(for displacement) order low-cut Bessel filters (Katsumata, 1993) are used in this study. The filters are recursive, and can be applied in real-time processing.

Several cutoff periods are used to measure $A$. Cutoff 


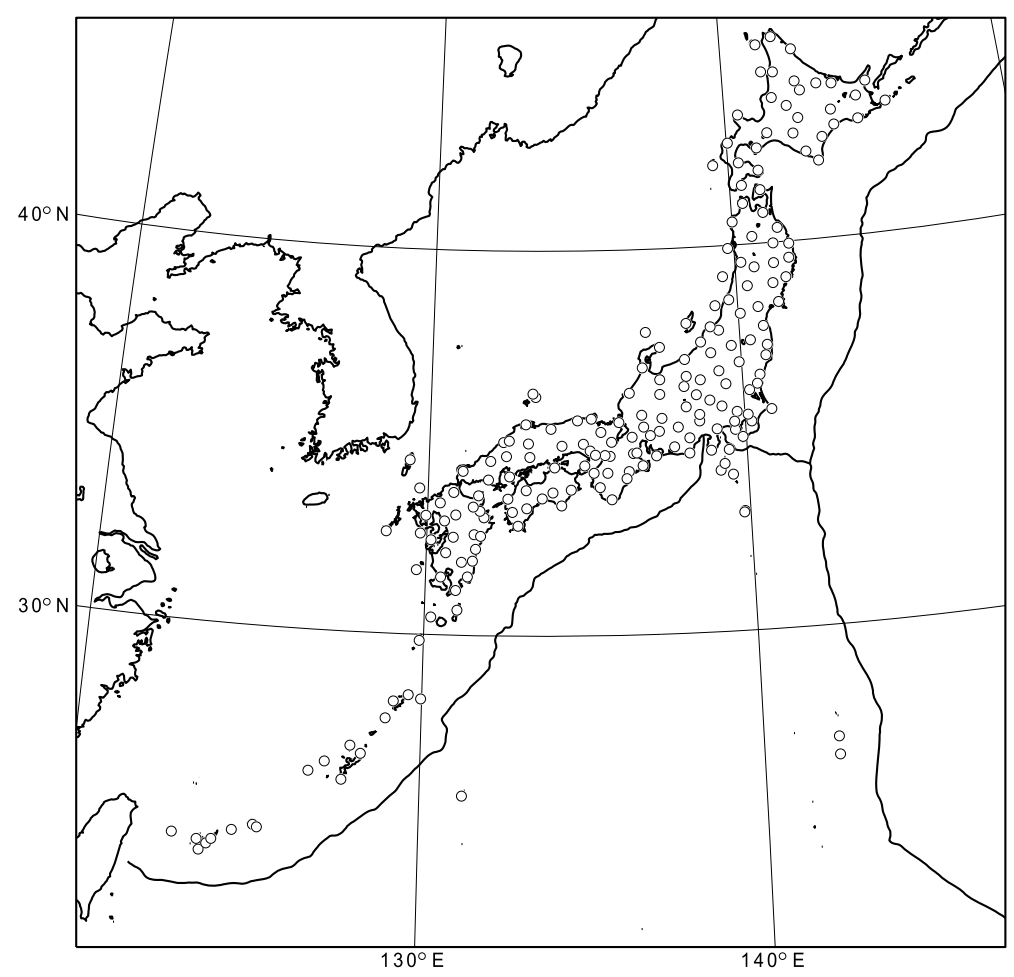

Fig. 3. Station map of the data used in this study. Circles denote the locations of the stations.

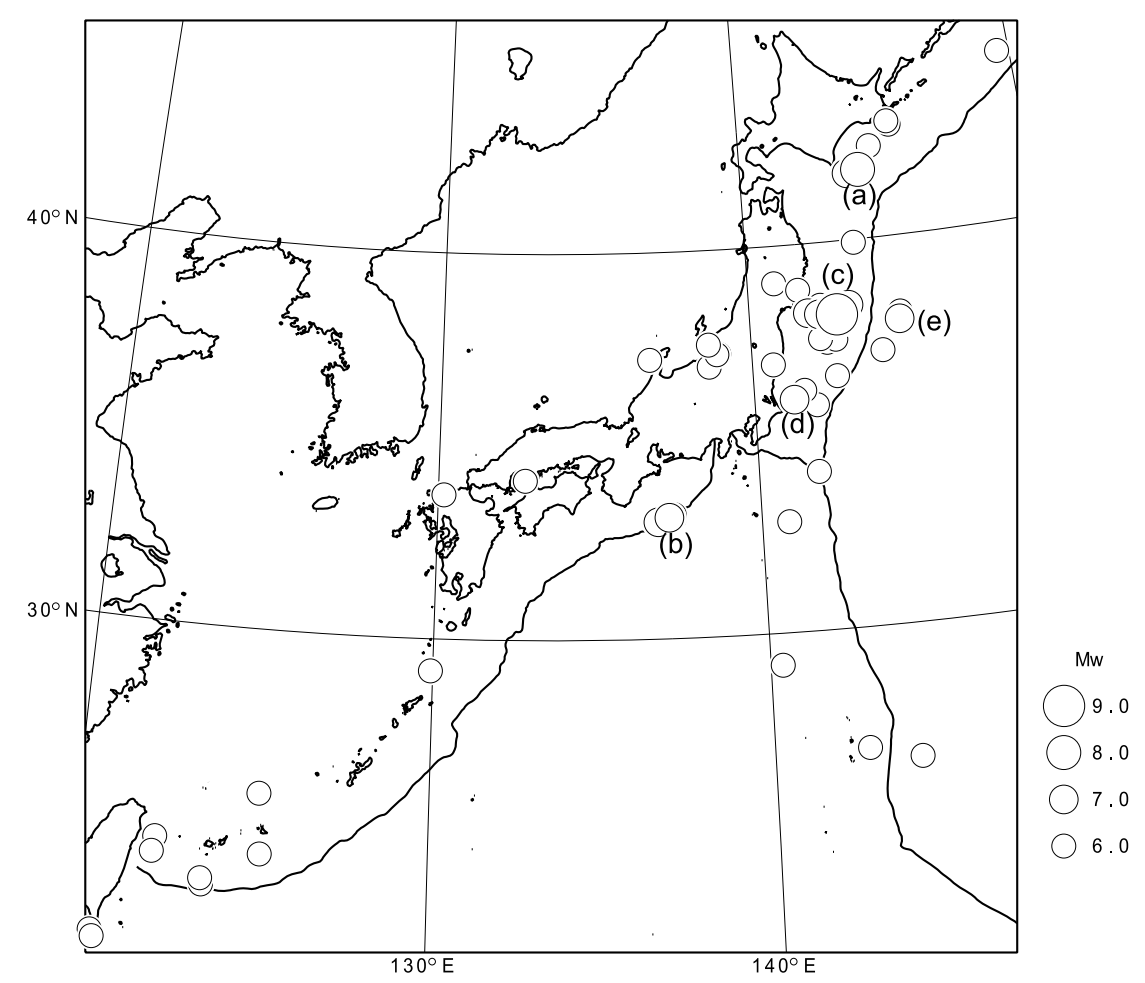

Fig. 4. Epicenters of the earthquakes for which the seismic records were obtained in this study. (a)-(e) indicate events in Fig. 9.

periods of low-cut filters are set at $1,2,5,10,20,50$, and 100 seconds. Various cutoff periods $\left(T_{c}\right)$ are used to accommodate a broard range of magnitudes. Examples of waveforms are presented in Fig. 2. Constants $a, b$ and $c$ are estimated so as to minimize the difference between $M$ in Eq. (1) and $M_{\mathrm{w}}$ of the Global CMT solutions. The constants in Eq. (1) are estimated for velocity/displacement and each cutoff period. We assume a common value of $a$ for all $T_{c}$ in Eq. (1), since we expect to see magnitude saturation in the result. The values of $b$ and $c$ are estimated for each $T_{c}$, individually.

Parameter $a$ is first estimated with data of earthquakes $M_{\mathrm{w}}>7$, then $b$ and $c$ are estimated with data including those of smaller events. When the constants $a, b$, and 
Table 1. Velocity magnitude determination coefficients in Eq. (1).

\begin{tabular}{rccc}
\hline$T_{c}(\mathrm{~s})$ & $a$ & $b$ & $c$ \\
\hline 1 & 1.43 & 4.08 & 1.18 \\
2 & 1.43 & 3.96 & 1.20 \\
5 & 1.43 & 3.68 & 1.64 \\
10 & 1.43 & 3.25 & 2.56 \\
20 & 1.43 & 2.81 & 3.60 \\
50 & 1.43 & 2.67 & 3.90 \\
100 & 1.43 & 2.47 & 4.39 \\
\hline
\end{tabular}

Table 2. Displacement magnitude determination coefficients in Eq. (1).

\begin{tabular}{rccc}
\hline$T_{c}(\mathrm{~s})$ & $a$ & $b$ & $c$ \\
\hline 1 & 1.23 & 3.48 & 3.02 \\
2 & 1.23 & 3.21 & 3.17 \\
5 & 1.23 & 2.61 & 4.10 \\
10 & 1.23 & 1.99 & 5.31 \\
20 & 1.23 & 1.46 & 6.39 \\
50 & 1.23 & 1.22 & 6.80 \\
100 & 1.23 & 1.24 & 6.64 \\
\hline
\end{tabular}

$c$ are estimated simultaneously, $M$ of great earthquakes $\left(M_{\mathrm{w}}>8\right)$ diverges further from $M_{\mathrm{w}}$. We adopt the value of $a$ for a $T_{c}$ which indicates the least dispersion for earthquakes including small ones, since such a value would be applicable to great earthquakes as well as to moderate ones.

The acceleration records were obtained with a seismic network installed by JMA. The station map is presented in Fig. 3. The accelerometers record ground motion up to \pm 3 $\mathrm{G}$ with a resolution of $0.5 \times 10^{-5} \mathrm{~m} / \mathrm{s}^{2}$ (Japan Meteorological Agency, 2011), which corresponds to a 22-bit resolution. We use only velocity and displacement amplitudes that exceed $0.5 \times 10^{-5} /\left(2 \pi / T_{c}\right) \mathrm{m} / \mathrm{s}$ and $0.5 \times 10^{-5} /\left(2 \pi / T_{c}\right)^{2}$ $\mathrm{m}$.

Records of fifty-five earthquakes of $M_{\mathrm{w}}>6.0$ from 2001 to 2011 are used. The epicenter map of the earthquakes is presented in Fig. 4.

\section{Results}

Tables 1 and 2 list the obtained values of coefficients $a$, $b$ and $c$ for the magnitude determination. We adopted the estimated $a$ for the cutoff period of fifty seconds, since it provided the smallest standard deviation for earthquakes, including smaller ones than those used for estimating $a$ $\left(M_{\mathrm{w}}>7\right)$. The coefficient for the amplitude $a$ in Eq. (1) is greater for velocity than for displacement. This relationship has been seen previously for short-period amplitude measurements (Watanabe, 1971; Katsumata, 2001).

Figures 5 and 6 present data plots with the fitted lines. The relationship between hypocentral distance and amplitude depends on the cutoff periods: the shorter the cutoff period, the steeper the attenuation. The displacement amplitude attenuation depends more on the cutoff period than does the velocity amplitude. The dependence on the period would be related to inelastic attenuation and the seismic wave type that exhibits a peak amplitude. Data deviates from the fitted line at short epicentral distances. The dispersion of $\log _{10} A$ ranges from $0.22^{2}$ to $0.33^{2}$ for the velocity magnitude, and from $0.21^{2}$ to $0.34^{2}$ for the displacement magnitude. Amplitude data of velocity and displacement have similar data dispersions.

Figures 7 and 8 illustrate the relationship between the moment magnitude $M_{\mathrm{w}}$ and the difference of the estimated magnitude $M$ (Eq. (1)) from $M_{\mathrm{w}}$. Since a common value of $a$ is used in Eq. (1), magnitude saturation is seen in shorter cutoff periods (Figs. 7 and 8). Standard deviations of $M-M_{\mathrm{w}}$ range from $0.18\left(T_{c}=100 \mathrm{~s}\right)$ to $0.32\left(T_{c}=1\right.$ $\mathrm{s})$ for velocity magnitude and from $0.15\left(T_{c}=100 \mathrm{~s}\right)$ to $0.27\left(T_{c}=1 \mathrm{~s}\right)$ for displacement magnitude. Deviations of velocity magnitudes are slightly larger than those of displacement magnitudes.

\section{Discussion}

\subsection{Velocity magnitude and displacement magnitude}

In this section, we will briefly discuss the suitability for tsunami warning of velocity and displacement magnitudes. Dispersion of data (Figs. 5 and 6) and differences from $M_{\mathrm{w}}$ (Figs. 7 and 8) do not clearly differ between velocity and displacement magnitudes. Data from accelerometers are used here with numerical integration, and data availability is limited by $a_{R} / \omega$ for velocity and $a_{R} / \omega^{2}$ for displacement, where $a_{R}$ is the sensor resolution of the accelerometer and $\omega$ is the angular frequency. Velocity magnitude is available for more events due to the limitation on the amplitude range.

For tsunami earthquakes such as the 1992 Nicaragua earthquake (Kanamori and Kikuchi, 1993), the lowfrequency component is more dominant than in normal earthquakes. The displacement magnitude is more sensitive to the low-frequency component than is the velocity magnitude. The displacement magnitude is thus preferable for tsunami warnings. The displacement magnitude is mainly examined in the following sections.

The integral of displacement is proportional to the seismic moment, and this might be better for tsunami warning than the displacement amplitude. However, accelerometers do not have enough resolution for more integration. When data of strong motion velocity meters are used for the same purpose, a longer-period component could be used. Since accelerometer networks are more dense than strong motion velocity meter networks, we use accelerometer data in this study.

\subsection{Application to rapid magnitude determination}

The proposed magnitude is considered to be used to observe the growth of magnitude value in real-time processing. Since recursive filters are used to obtain the velocity/displacement records and the transmission and processing delay could be no more than several seconds, it is possible to see the magnitude value change soon after the hypocenter determination. Because they are not used, inversion analysis and phase identification do not introduce additional delay.

The proposed magnitude determination method is applied to some large earthquakes, and the results are presented in Fig. 9. The horizontal axis in the figure represents time from the earthquake occurrence. The magnitude is determined from maximum amplitudes measured until the time indicated on the horizontal axis at the closest three to ten stations. The magnitude value changes with the arrivals of larger seismic waves. It reaches a stable value when peak 

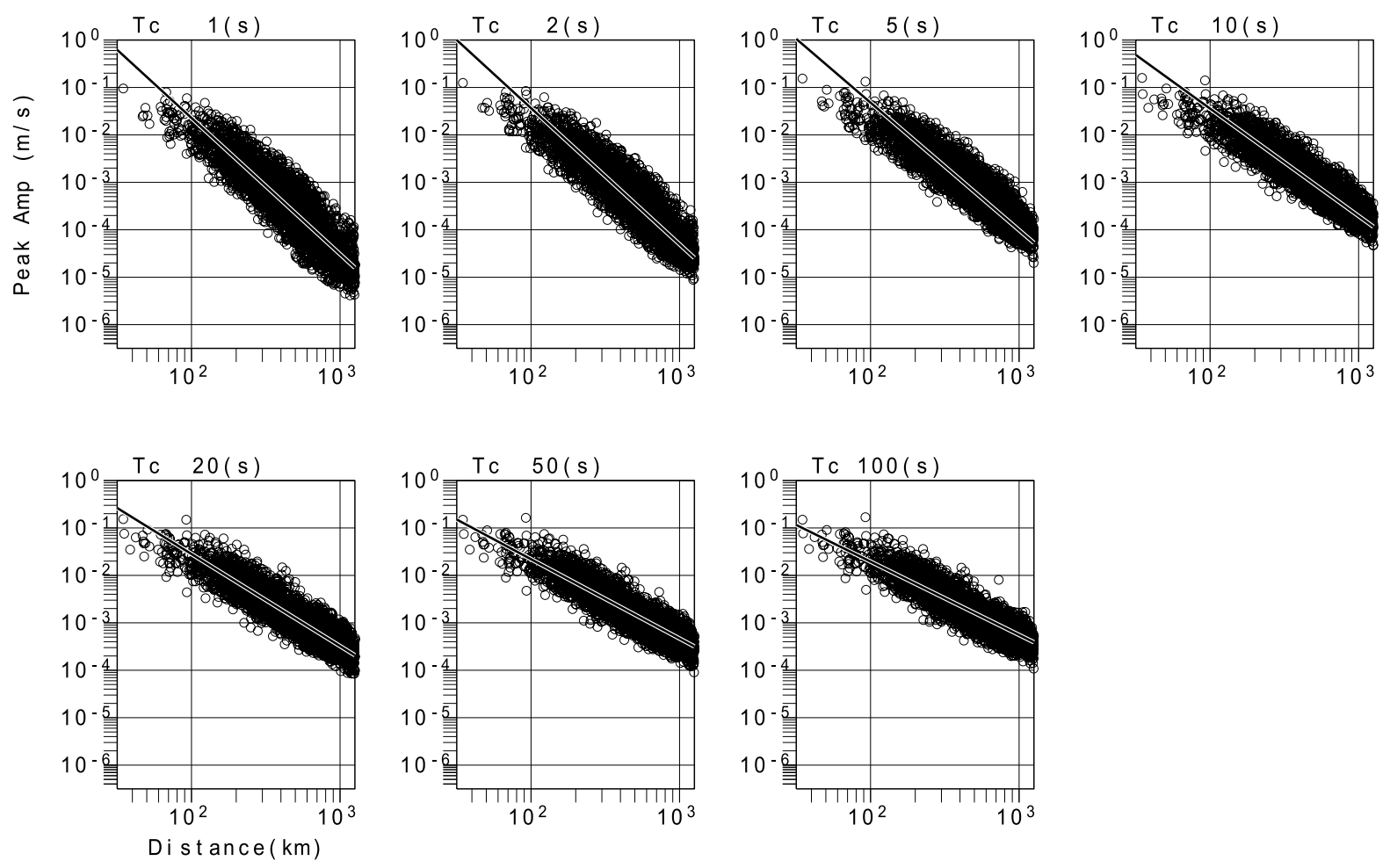

Fig. 5. Relationship between hypocentral distance and velocity amplitude. The line denotes the fitted relationship (Eq. (1)). The data plots are shifted in peak amplitude by $\left(7-M_{\mathrm{w}}\right) / a$ to adjust magnitude differences.
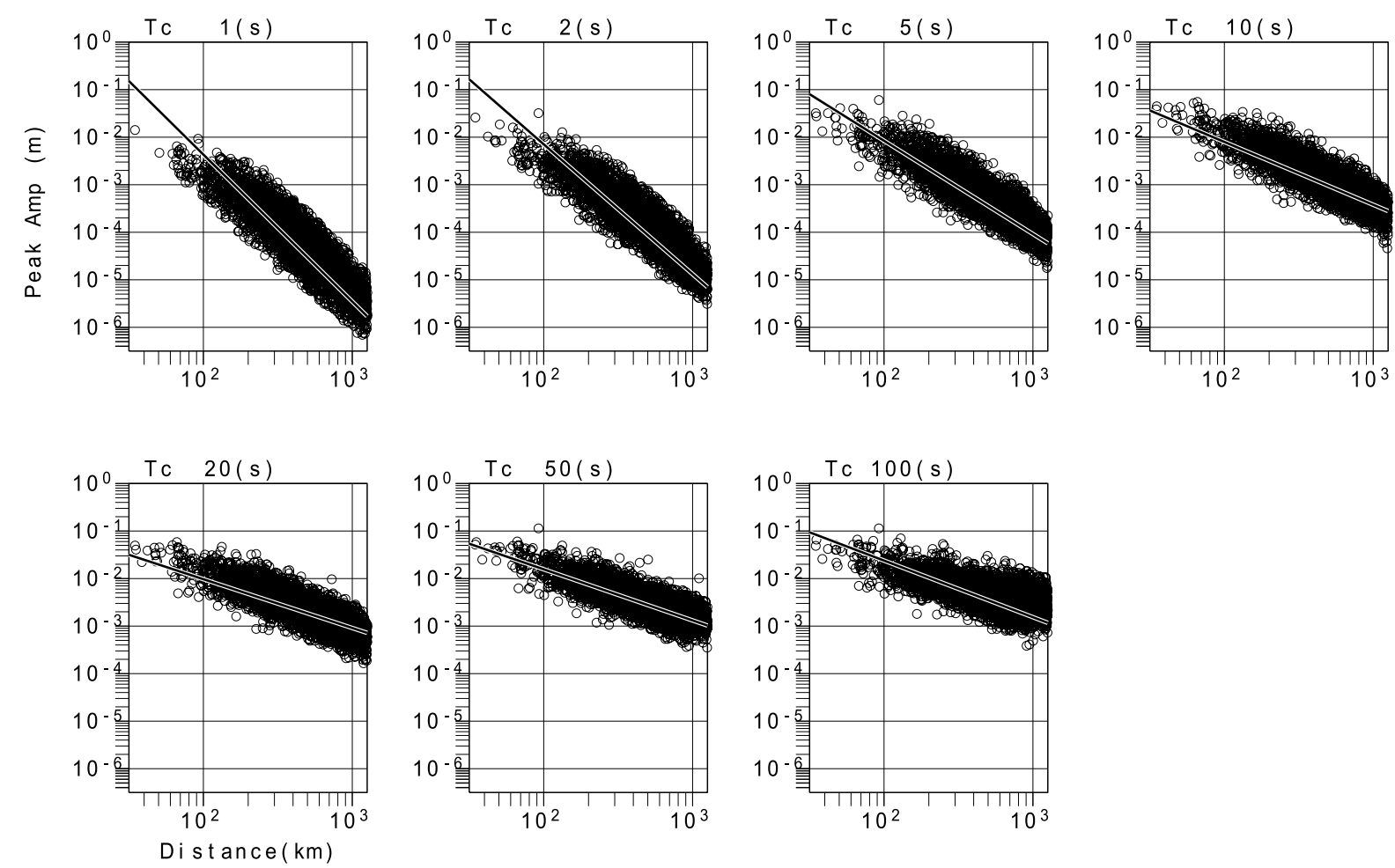

Fig. 6. Relationship between hypocentral distance and displacement amplitude. The line denotes the fitted relationship (Eq. (1)). The data plots are shifted in peak amplitude by $\left(7-M_{\mathrm{w}}\right) / a$ to adjust magnitude differences.

amplitudes are observed at all of the closest ten stations. The epicenters of the events in Fig. 9 are labeled (a)-(e). Event (f) is an earthquake off the coast of Chile in 2010. Data obtained by the University of Chile are used for event (f).
For the 2011 off the Pacific coast of Tohoku earthquake, the magnitude reached the final value within 140 seconds. The target time of the first tsunami warning in JMA is three minutes, so 140 seconds is a satisfactory time for the first tsunami warning. The final magnitude was 8.8 in the figure, 

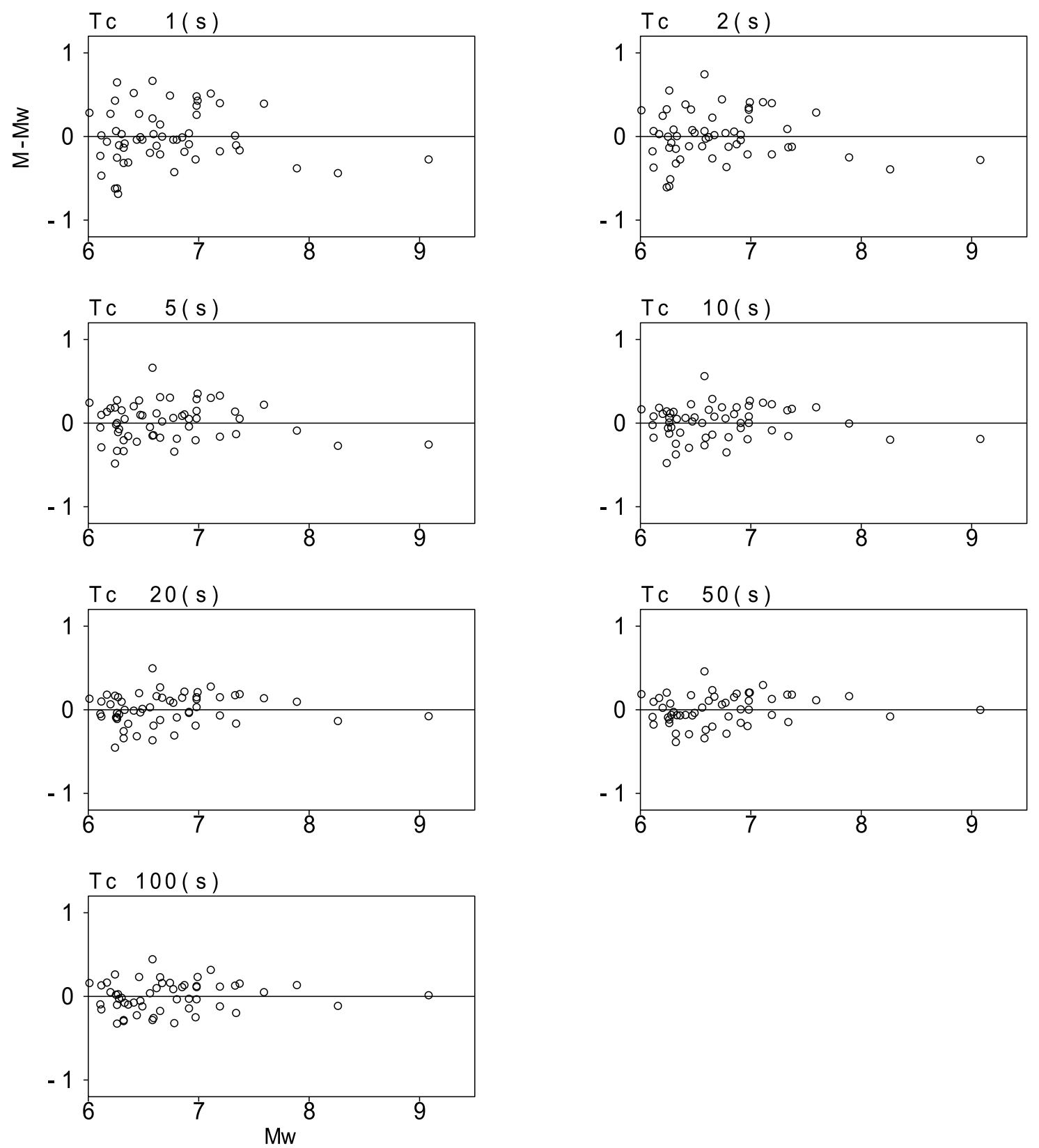

Fig. 7. Difference between magnitude determined from the peak velocity of various cutoff periods $\left(T_{c}\right)$ and the moment magnitude.

which is less than $M_{\mathrm{w}} 9.1$.

The time to reach the final magnitude of other earthquakes is less than three minutes. The times are delayed for events with epicenters far from the closest stations such as earthquakes off the Kuril Islands. However, tsunami arrivals at the nearest coasts would also be delayed for those earthquakes.

As expected, magnitude saturation is observed in Fig. 9. Magnitudes of shorter cutoff periods are generally smaller than those of longer cutoff periods. Differences among $M_{20}$, $M_{50}$ and $M_{100}$ are not so large, but the difference between $M_{10}$ and $M_{20}$ is relatively large (the subscript denotes the cutoff period). Two large pulsed peaks with widths of about twenty seconds are seen in the seismic records in Fig. 2. The asperity size and its slip process would have defined the pulse width which is related to the characteristics of the magnitude saturation. The similarity of $M_{20}, M_{50}$ and
$M_{100}$ might be related to fault-rupture characteristics of the regions.

For the 2010 Chile event, short-period magnitudes are greater than long-period magnitudes. This reversed magnitude relationship would be related to the concentrated distribution of the used stations in the northern region of the source area, the relatively southern location of the epicenter, and a large slip in the northern area (Lay et al., 2010). Since the amplitude decay is steeper in short-period magnitudes than in long-period magnitudes (Fig. 6), the uneven station distribution and the improper assumption of the distance to the source affect the short-period magnitudes more. Even in such a case, the long-period magnitude is considered to be more reliable than the short-period magnitude.

The time to reach the final value and the final magnitude depend on the station distribution and the upper limit of station numbers. Figure 10 presents variations of estimated 

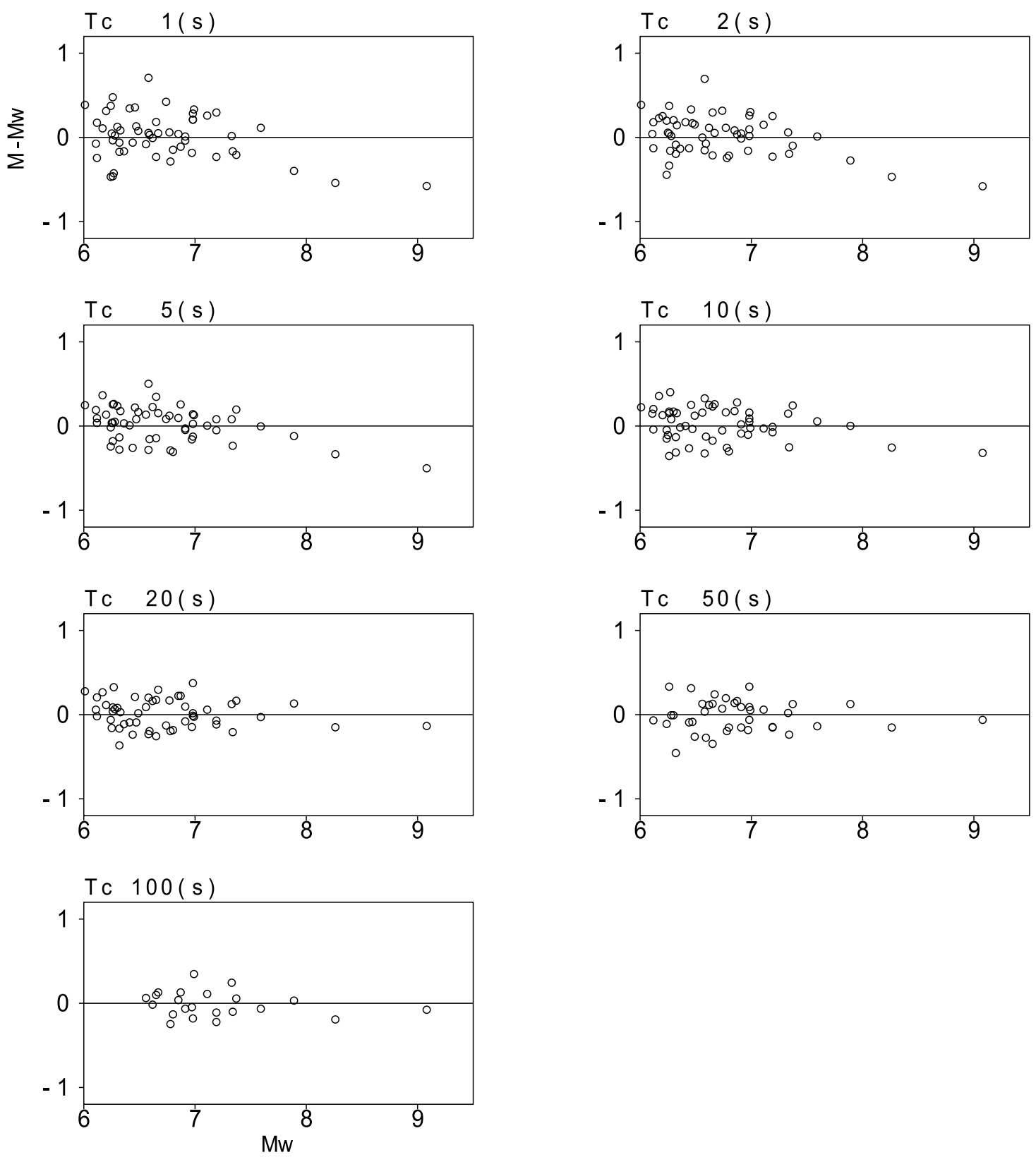

Fig. 8. Difference between magnitude determined from the peak displacement of various cutoff periods $\left(T_{c}\right)$ and the moment magnitude.

Table 3. Velocity structure model used to calculate synthetic records for Fig. 12.

\begin{tabular}{cccccr}
\hline $\begin{array}{c}\text { Depth (Top) } \\
\mathrm{km}\end{array}$ & $\begin{array}{c}\text { Velocity }(P) \\
\mathrm{km} / \mathrm{s}\end{array}$ & $\begin{array}{c}\text { Velocity }(S) \\
\mathrm{km} / \mathrm{s}\end{array}$ & $\begin{array}{c}\text { Density } \\
\mathrm{g} / \mathrm{cm}^{3}\end{array}$ & $Q_{P}$ & $Q_{S}$ \\
\hline 0 & 3.0 & 1.44 & 2.3 & 150 & 75 \\
1 & 5.0 & 2.90 & 2.55 & 300 & 150 \\
4 & 6.3 & 3.60 & 2.75 & 400 & 200 \\
12 & 7.1 & 3.95 & 2.95 & 400 & 200 \\
25 & 7.8 & 4.30 & 3.15 & 400 & 200 \\
\hline
\end{tabular}

magnitudes with the period from the event origin time for various upper limits of station numbers for the 2011 off the Pacific coast of Tohoku earthquake. As expected, the magnitude reaches the final value earlier for fewer upper limit stations. However, the time difference in reaching the final value is not large in Fig. 10. The final magnitude increases with more stations, and approaches $M_{\mathrm{w}}(9.1)$. No large dif- ference in dispersion is seen. The upper limit station number can be set arbitrarily, and could be determined based on the required time limit and the difference from the final value.

The magnitudes obtained in this study are compared with $M_{\mathrm{wp}}$ (Tsuboi et al., 1995; Whitmore et al., 2002) in Fig. 11 for the 2011 off the Pacific coast of Tohoku earthquake. 

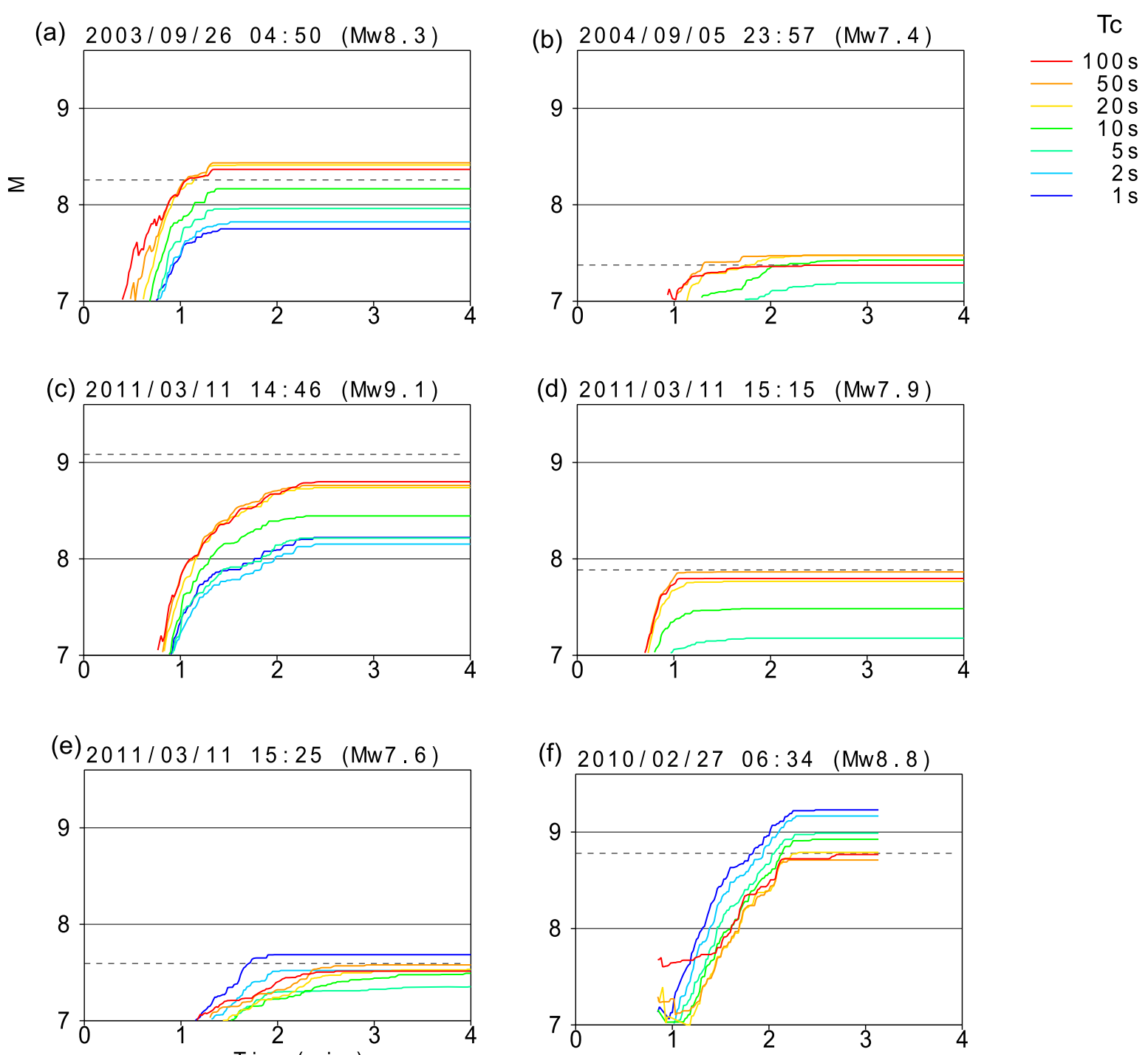

Fig. 9. Variation of displacement magnitudes versus elapsed time from the event origin time. The magnitudes are calculated from data acquired at the closest three to ten stations. The colors indicate the periods of the low-cut filters. Epicenters (a)-(e) are presented in Fig. 4 . The origin times are local times except for event (f).

Station magnitudes are shown in the figure with the time when the peak amplitudes were measured on the horizontal axis. A short peak-time generally means a short epicentral distance. Both magnitudes are determined from data of strong motion velocity meters installed by the National Research Institute for Earth Science and Disaster Prevention. The instrumental response is corrected with the recursive deconvolution filter proposed by Kanamori and Rivera (2008). Since the seismic wave for $M_{\mathrm{wp}}$ is restricted to the period between $P$ and $S$ arrivals, and $T_{S}-T_{P}$ at a station of short epicentral distance is less than the source duration, the resultant $M_{\text {wp }}$ at a close station is much less than the moment magnitude of the event $\left(M_{\mathrm{w}} 9.1\right) . M_{\mathrm{wp}}$ at stations become stable after about four minutes from the event origin time. On the other hand, the magnitude of this study scatter around the magnitude 9 even at close stations. However, the station magnitude scatter of this study seems to be larger than that of $M_{\mathrm{wp}}$.

\subsection{Effect of fault type}

Events of the same size with a different focal mechanism radiate seismic waves of different amplitude. The amplitude difference due to the fault type is investigated with synthetic records, and the results for dip and strike slip events are shown in Fig. 12. The synthetic records are calculated with the method of Takeo (1985), assuming a velocity structure in Table 3, a focal depth of $20 \mathrm{~km}$, and a triangle source time function of sixty second duration. A high-pass filter of 100 -s cutoff is applied to the records.

The amplitude reduces considerably when the station is located in the direction of the nodal planes. Since the compression/tension axis is usually oriented normal to the trench axis for events around a convergent plate boundary and the stations are installed in inland areas, it is considered that the observed amplitude would not become so small for local events. When seismic waves from events near Kuril Islands are observed on the Japan Islands, the stations are distributed around the direction of the null axis of the events and the magnitude would be underestimated. For the strike- 


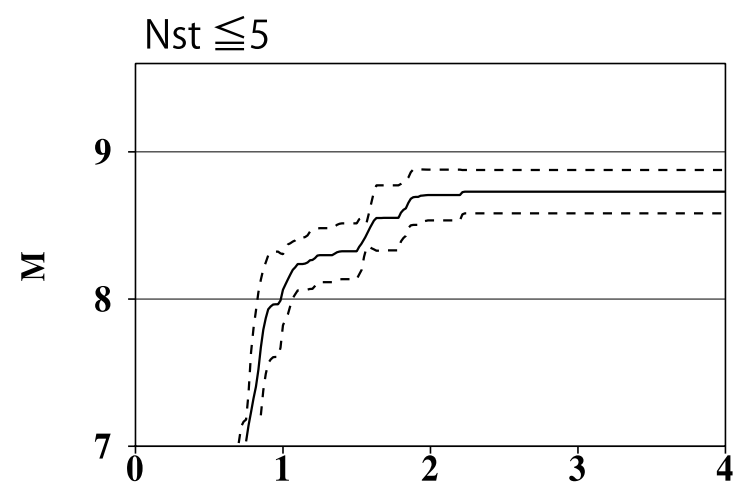

Nst $\leqq 15$

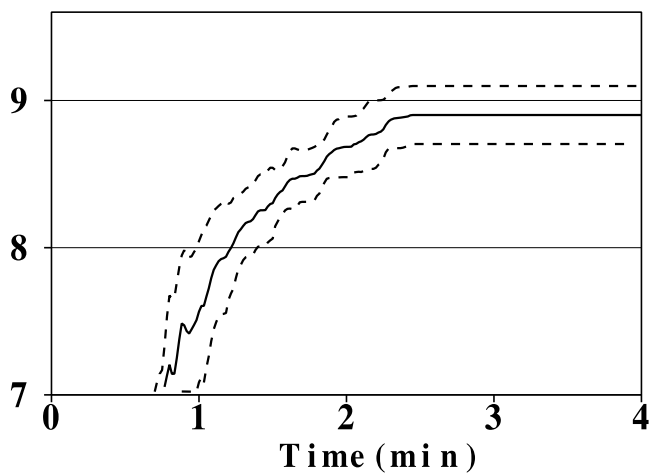

Nst $\leqq 10$

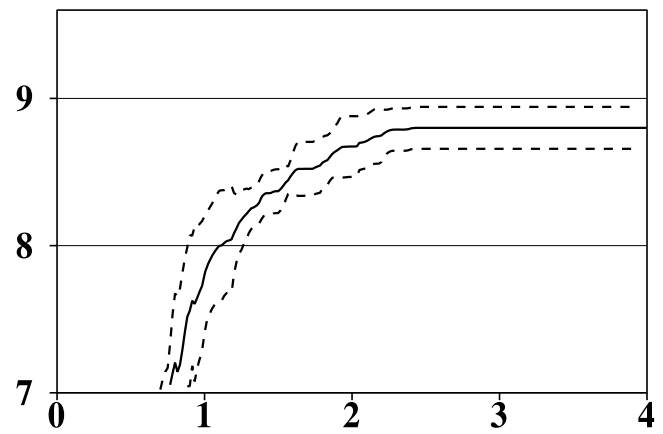

Nst $\leqq 20$

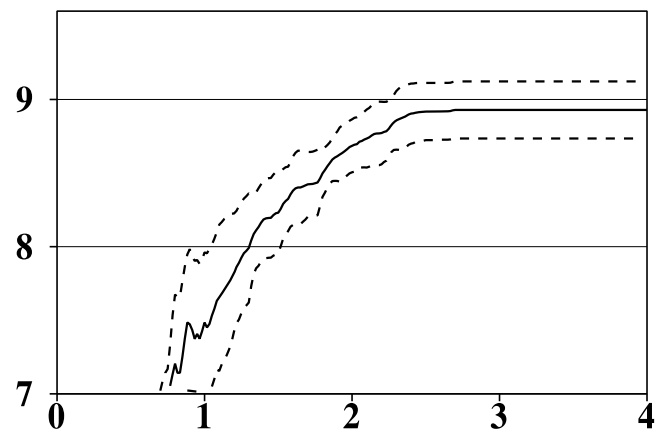

Fig. 10. Variation of displacement magnitudes calculated for various upper limit station numbers $\left(N_{\text {st }}\right)$ for the 2011 off the Pacific coast of Tohoku earthquake. The low cutoff period is set at 100 (s). The horizontal axis denotes the elapsed time from the event origin time. The broken curves indicate the \pm standard deviation.

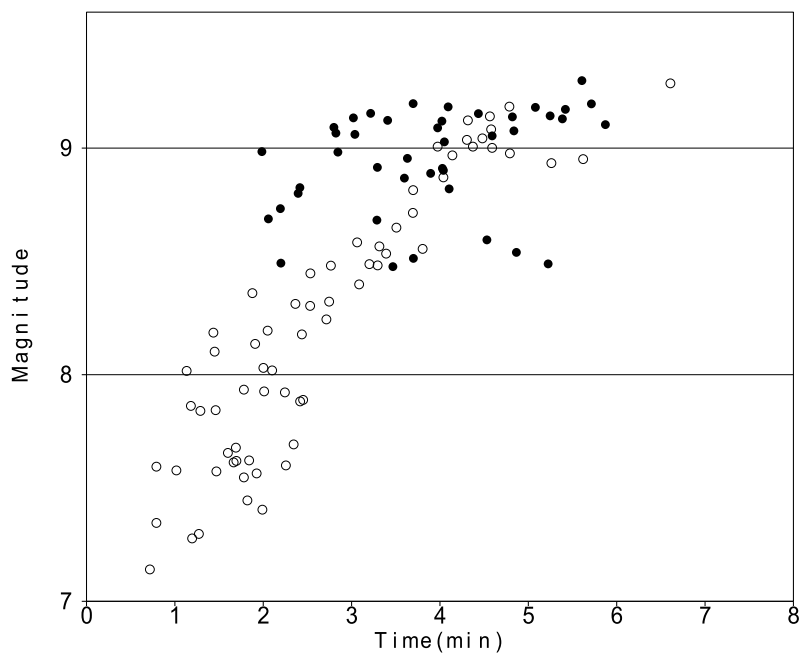

Fig. 11. Comparison between $M_{\mathrm{wp}}$ (Tsuboi et al., 1995; Whitmore et al., 2002) (the open circles) and the magnitude of this study (the solid circles, $T_{c}=100 \mathrm{~s}$ ) for the 2011 off the Pacific coast of Tohoku earthquake. Each point denotes a station magnitude, and the horizontal axis shows the arrival time of the peak amplitude from the event origin time. The amplitude search range for $M_{\mathrm{wp}}$ is restricted within $P$ and $S$ arrivals.

slip event, the averaged amplitude is a little smaller $(\times 0.8)$ than that for the dip-slip event in Fig. 12.

\subsection{Dependence on the epicenter location}

The source region of the 2011 off the Pacific coast of Tohoku earthquake extended about $450 \mathrm{~km}$ (Yoshida et al.,

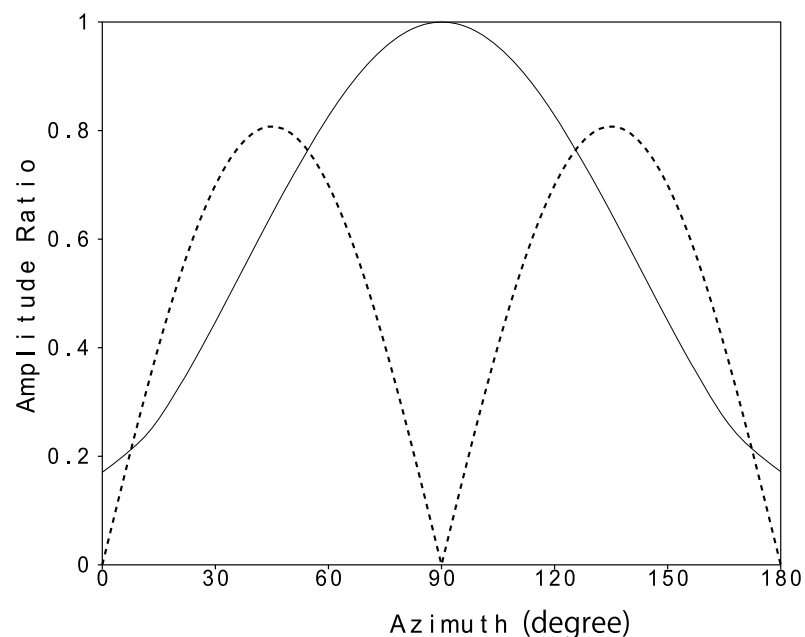

Fig. 12. Normalized vertical amplitude of synthetic waves for different fault types. The solid curve denotes amplitude for a dip slip event of strike 0 (degree), dip 20 (degrees), rake 90 (degrees), and a depth of 20 $\mathrm{km}$ measured at $300 \mathrm{~km}$ of epicentral distance and an azimuth from 0 to 180 (degrees). The broken curve denotes the amplitude for a strike slip event of strike 0 (degree), dip 90 (degrees), and rake 0 (degrees), and the same focal depth at the same epicentral distance.

2011). An earthquake of a different epicenter might have a similar size of source region. Different epicenters are assumed for the magnitude determination. Figure 13 presents magnitude differences for different epicenters for a 100 -s cutoff period. Data acquired at the closest ten stations are used. The reference magnitude is that for the estimated 


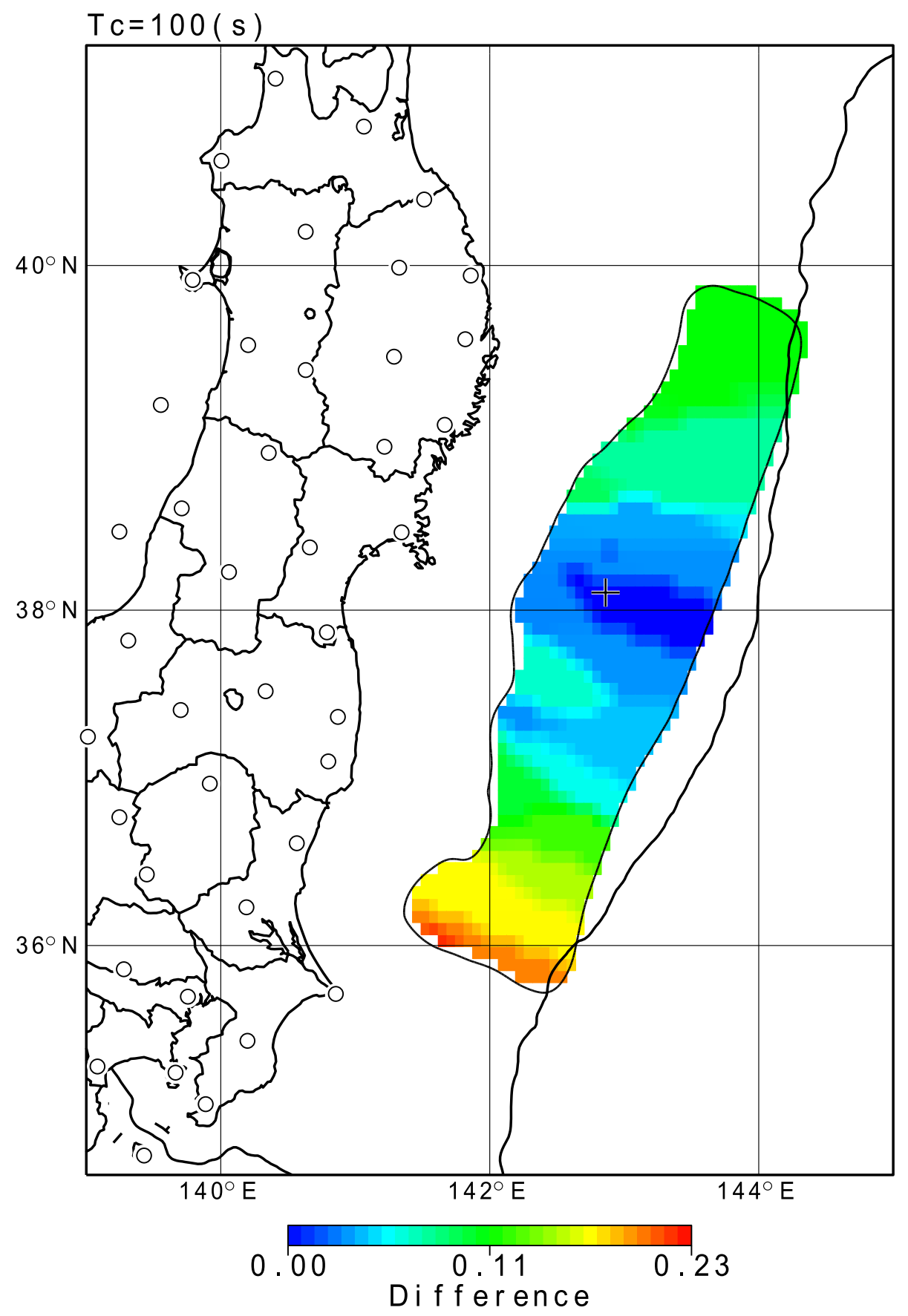

Fig. 13. Differences of displacement magnitude for assumed epicenters from that for the estimated epicenter (the cross) of the 2011 off the Pacific coast of Tohoku earthquake. The closest ten stations to the assumed epicenter are used to calculate the magnitude. Epicenters are assumed inside a contour of the 5-meter slip estimated by Yoshida et al. (2011). The circles indicate the station locations.

hypocenter (the cross in the figure). The epicenters are assumed to be within the 5-m-slip contour of Yoshida et al. (2011). The magnitude difference is less than 0.25 of the magnitude unit. The difference does not exceed 0.1 over a large part of the area, so the magnitude difference is not significant.

The difference is greater for magnitudes of shorter periods due to high amplitude decay rates of short-period magnitudes (Fig. 5).

\section{Conclusions}

A rapid magnitude determination method for tsunami warning based on the peak velocity and displacement of long-period seismic waves is presented. Cutoff periods up to 100 seconds were used to obtain the seismic wave data from acceleration records. The magnitude did not saturate up to magnitude 9 and could be determined within three minutes for the great earthquake on March 11, 2011, off the Tohoku District, Japan.

Acknowledgments. We used data obtained by the Japan Meteorological Agency, University of Chile, and National Research Institute for Earth Science and Disaster Prevention. We used 
programs developed by Dr. Takeo to calculate synthetic records. We greatly appreciate kind and thoughtful comments from two anonymous reviewers. This study was partly supported by the SATREPS project of "Enhancement of technology to develop tsunami-resilient community".

\section{References}

Aki, K., Scaling law of seismic spectrum, J. Geophys. Res., 72, 1217-1231, 1967.

Hara, T., Magnitude determination using duration of high frequency energy radiation and displacement amplitude: Application to tsunami earthquakes, Earth Planets Space, 59, 561-565, 2007.

Hirose, F., K. Miyaoka, N. Hayashimoto, T. Yamazaki, and M. Nakamura, Outline of the 2011 off the Pacific coast of Tohoku Earthquake $\left(M_{\mathrm{w}}\right.$ 9.0)-Seismicity: foreshocks, mainshock, aftershocks, and induced activity_, Earth Planets Space, 63, 513-518, 2011.

Japan Meteorological Agency, Strong motion observation report 2010 (CD-ROM), Japan Meteorological Agency, Tokyo, 2011.

Kamigaichi, O., JMA earthquake early warning, J. Jpn. Assoc. Earthq. Eng., 4, 134-137, 2004.

Kanamori, H. and M. Kikuchi, The 1992 Nicaragua earthquake: a slow tsunami earthquake associated with subducted sediments, Nature, 361, 714-716, 1993.

Kanamori, H. and L. Rivera, Source inversion of W phase: speeding up seismic tsunami warning, Geophys. J. Int., 175, 222-238, 2008.

Katsumata, A., Automatic designing of Bessel digital filters, Quart. J. Seismol., 56, 17-24, 1993 (in Japanese).

Katsumata, A., Relationship between displacement and velocity amplitudes of seismic waves from local earthquakes, Earth Planets Space, 53, 347-355, 2001

Katsumata, A., Revision of the JMA displacement magnitude, Quart. J. Seismol., 67, 1-11, 2004 (in Japanese with English abstract).

Lay, T., C. J. Ammon, H. Kanamori, K. D. Koper, O. Sufri, and A. R. Hutko, Teleseismic inversion for rupture process of the 27 February 2010 Chile (Mw 8.8) earthquake, Geophys. Res. Lett., 37, L13301, doi:10.1029/2010GL043379, 2010 .
Lomax, A. and A. Michelini, $M_{w p d}$ : A duration-amplitude procedure for rapid determination of earthquake magnitude and tsunamigenic potential from P Waveforms, Geophys. J. Int., 176, 200-214, doi:10.1111/j.1365-246X.2008.03974.x, 2009.

Takeo, M., Near-field synthetic seismograms taking into account the effects of anelasticity-The effects of anelastic attenuation on seismograms caused by sedimentary layer-, Pap. Meteorol. Geophys., 36, 245-257, 1985 (In Japanese with English abstract).

Tsuboi, S., K. Abe, K. Takano, and Y. Yamanaka, Rapid determination of Mw from broadband P waveforms, Bull. Seismol. Soc. Am., 85, 606$613,1995$.

Watanabe, H., Determination of earthquake magnitude at regional distance in and near Japan, Zisin II, 24, 189-200, 1971 (in Japanese with English abstract).

Whitmore, P. M., S. Tsuboi, B. Hirshorn, and T. J. Sokolowski, Magnitudedependent correction for $M_{w p}$, Sci. Tsunami Haz., 20, 187-192, 2002.

Wu, Y. M., T. C. Shin, and Y. B. Tsai, Quick and reliable determination of magnitude for seismic early warning, Bull. Seismol. Soc. Am., 88, 1254-1259, 1998.

Wu, Y. M. and L. Zhao, Magnitude estimation using the first three seconds P-wave amplitude in earthquake early warning, Geophys. Res. Lett., 33, L16312, doi:10.1029/2006GL026871, 2006.

Yoshida, Y., Magnitude determination from P-wave amplitude $\left(M_{P}\right), J M A$ Tech. Note Seismol. Volcanol., 71, 41-52, 1995.

Yoshida, Y., H. Ueno, D. Muto, and S. Aoki, Source process of the 2011 off the Pacific coast of Tohoku Earthquake with the combination of teleseismic and strong motion data, Earth Planets Space, 63, 565-569, 2011.

Zollo, A., M. Lancieri, and S. Nielsen, Earthquake magnitude estimation from peak amplitudes of very early seismic signals on strong motion records, Geophys. Res. Lett., 33, L23312, doi:10.1029/2006GL027795, 2006.

A. Katsumata (e-mail: akatsuma@mri-jma.go.jp), H. Ueno, S. Aoki, Y. Yoshida, and S. Barrientos 